\title{
Influencia de las masas boscosas en el régimen hídrico de una cuenca semiárida, Chile
}

\author{
Influence of the wooded masses on hydric regime in a semiarid river basin, Chile
}

\author{
ROBERTO PIZARRO T ${ }^{1}$, ANDRES BENITEZ G. ${ }^{2}$, CESAR FARIAS D. ${ }^{1}$, CRISTIAN JORDAN D. ${ }^{1}$, \\ FERNANDO SANTIBAÑEZ Q. ${ }^{3}$, CLAUDIA SANGÜESA P. ${ }^{1}$, JUAN PABLO FLORES V. ${ }^{1}$, \\ ENZO MARTINEZ A. ${ }^{1}$, LEONARDO ROMAN A. ${ }^{1}$ \\ ${ }^{1}$ Depto. Gestión Forestal y Ambiental, Universidad de Talca, Casilla 721, Talca. Chile. \\ E-mail: rpizarro@utalca.cl \\ ${ }^{2}$ Carmencita $\mathrm{N}^{\circ} 246$ Depto. 62, Las Condes, Santiago. Chile. \\ ${ }^{3}$ Centro Agrimed, Facultad de Ciencias Agrarias y Forestales, Universidad de Chile, Santiago. Chile.
}

\begin{abstract}
SUMMARY
This article analyzes the influence which they have the wooded masses in the water production of the river basin of the Purapel river, located in the central zone of Chile. This river basin has a surface of 264., $\mathrm{km}^{2}$ and it has been affected by processes of vegetational substitution, happening of native forests a massive forestation, mainly of Pinus radiata (D.Don). The made multitemporary analysis, showed that the vegetal cover I vary of 0 per cent of forest plantations in 1957, 51,7 per cent for year 1997. The methodology studied the use of the ground in 1957, 1978 and 1997; the precipitations were analyzed and the flow and hidrogramas of hour in hour studied; the variation of the water reserves was analyzed on monthly scale and annual, the runoff coefficients of monthly and annual were analyzed, the maximum flow, and finally, their relation with the vegetal cover and the water production studied between years 1960 and 2000. The results allow to conclude that for the river basin of the Purapel river, differences in the observed hydrology behavior in the 40 studied years do not exist. The oscillations observed in the hydrologyc variables would have to the amounts and happened intensities of precipitation and to the high intermonthly variability. Only the monthly reserves show a different behavior, since they tend to be increased in 1979/2000 period. It would have to important precipitations fallen in the decade of the 80 , and not directly to a change of the type of vegetal cover.
\end{abstract}

Keywords: runoff-vegetation relationship, precipitation, flow, reserves, surface runoff coefficient, maximum flow.

\section{RESUMEN}

Este artículo analiza la influencia que tienen las masas boscosas en la producción de agua de la cuenca del río Purapel, ubicada en la zona central de Chile. Esta cuenca tiene una superficie de $264,6 \mathrm{~km}^{2}$ y se ha visto afectada por procesos de sustitución vegetacional, pasando de bosques nativos a una forestación masiva, principalmente de la especie Pinus radiata (D. Don). El análisis multitemporal realizado, mostró que la cobertura vegetal varió del $0 \%$ de plantaciones forestales en el año 1957, al 51,7\% para el año 1997. La metodología estudió el uso del suelo en los años 1957, 1978 y 1997; se analizaron las precipitaciones y se estudiaron los caudales y los hidrogramas de hora en hora; se analizó la variación de las reservas de agua a escala mensual y anual, se analizaron los coeficientes de escorrentía mensual y anual, se estudiaron los caudales máximos, y finalmente, su relación con la cobertura vegetal y la producción de agua entre los años 1960 y 2000. Los resultados permiten concluir que para la cuenca del río Purapel, no se observan diferencias estadísticas en las cantidades de agua generadas por los escurrimientos en los 40 años estudiados. Las oscilaciones observadas en las variables hidrológicas se deberían a los montos e intensidades de precipitación ocurridos y a la alta variabilidad intermensual. Sólo las reservas mensuales muestran un comportamiento distinto, ya que tienden a incrementarse en el periodo 1979/2000. Ello se debería a las importantes precipitaciones caídas en la década del 80 , y no directamente a un cambio del tipo de cobertura vegetal.

Palabras clave: relación precipitación-escorrentía-vegetación, precipitación, caudal, reservas, coeficiente de escorrentía, caudales máximos. 


\section{INTRODUCCION}

La influencia de la vegetación en el proceso precipitación-escorrentía es una relación que se ha estudiado desde hace más de 100 años, pero desde la segunda mitad del siglo XX es cuando se ha avanzado de manera más importante. Así, por ejemplo, se ha estudiado la influencia de la vegetación y las masas boscosas sobre la infiltración del agua en el suelo, en donde el continuo aporte de materia orgánica origina una estructura más granular de éste, que actúa favoreciendo la infiltración (1). Además, existe consenso en que el impacto de los bosques sobre la escorrentía consiste en la modificación de la forma en que estos volúmenes acceden a los cauces, disminuyendo drásticamente las aportaciones superficiales y aumentando las aportaciones subterráneas $(2,3)$.

Experiencias en España indican que el bosque genera un ecoclima particular en comparación con el de un sitio descubierto, dado que al interior de éste se produce una reducción de la luminosidad de hasta un $90 \%$, disminuyendo la temperatura media anual en unos $4^{\circ} \mathrm{C}$. Además, se reduce la velocidad del viento a la cuarta parte y se presentan condiciones de mayor humedad con un aumento medio del orden del $10 \%$ diario (4). Se sabe que la vegetación afecta a las precipitaciones orográficas, donde los bosques acrecientan la altura efectiva que deben remontar los vientos, traduciéndose en un aumento de las precipitaciones que va entre un $0,8 \%$ y un $1 \%$, según experiencias centroeuropeas. Además, el efecto de fricción que realizan los bosques tiende a frenar la velocidad de avance de los vientos, favoreciendo el movimiento ascensional de éstos; según datos experimentales, se produciría un aumento de un 2 a un $3 \%$ de las precipitaciones (3).

El análisis del ciclo hidrológico en cuencas francesas muestra que la destrucción de la cobertura vegetal hace que se incremente la escorrentía superficial en $150 \mathrm{~mm}$ (5). Un estudio en esta temática hecho por Calder (6), que analizó 94 cuencas en todo el mundo, afirma que en términos generales, un incremento de un $10 \%$ de la cobertura forestal en bosques de pinos y eucaliptos, determina una baja de $40 \mathrm{~mm}$ en el caudal anual. Además, señala que una forestación de un $10 \%$ de una zona previamente cubierta por pastos, determina que la escorrentía anual disminuya entre 10 y $25 \mathrm{~mm}$. Otro estudio a nivel de balance de agua en cuencas de condiciones templadas y semiáridas, concluye que las características del perfil de suelo (permeabilidad y capacidad de almacenaje de agua), la cobertura vegetal y las condiciones de clima (precipitación y evaporación potencial), son las variables que más influyen en el balance de agua (7).

Dentro de este mismo contexto, muchos autores coinciden en que el principal efecto que tienen las plantaciones forestales sobre el balance hídrico es la cantidad de precipitación que es retenida por el dosel (pérdidas de agua por intercepción) y que, por lo tanto, se reduce su participación en la economía hídrica de estos ecosistemas $(8,9,10,11)$. En este contexto, Huber e Iroumé (12) estudiaron los procesos de intercepción de precipitaciones en 29 ensayos, con distintos tipos de cobertura arbórea (bosque nativo y plantaciones forestales) establecidos entre la VIII y la $\mathrm{X}$ Regiones de Chile. Esta investigación estableció que las plantaciones presentan retenciones de precipitación entre el 18 y $35 \%$, mientras que el bosque nativo presenta intercepción entre el 14 y 40\%, siendo las pérdidas por intercepción en promedio mayores en las plantaciones forestales que en los bosques nativos.

Las investigaciones en este tema han llevado a determinar que el agua consumida por bosques adultos de Pinus radiata (D. Don) por concepto de evapotranspiración, supera en un $80 \%$ a la consumida por praderas (13). De esta forma, la forestación también modifica la cantidad de agua involucrada en la evaporación desde el suelo y la involucrada por transpiración de los árboles (evapotranspiración neta). Este valor depende del total de agua disponible en el suelo, las características de las plantaciones y las condiciones meteorológicas $(11,14)$.

La cantidad de precolación de un suelo está influida por la velocidad de infiltración, la capacidad de almacenamiento de agua en el suelo y del valor residual entre las precipitaciones y la evapotranspiración $(11,15,16)$. De esta manera, está regulada por variables que influyen sobre la cantidad que alcanza el suelo y la evapotranspiración neta.

Junto con producir efectos importantes sobre las precipitaciones, principalmente en lo referido a pérdidas por intercepción, evapotranspiración y precolación, la presencia de plantaciones forestales provoca cambios relevantes en la escorrentía, 
manifestándose su influencia tanto en la escorrentía superficial, como en las reservas de agua, así como también en los caudales máximos y mínimos. Así, los bosques disminuyen drásticamente las aportaciones de agua superficial, la velocidad de la escorrentía y el caudal punta de descarga (17), debido a tasas más altas de evaporación e intercepción, lo cual es particularmente sensible cuando se comparan plantaciones de rápido crecimiento, frente a cubiertas precedentes de pastos y/o arbustos $(2,5,6,18,19,20,21,22,23)$.

La sustitución o eliminación de una cubierta vegetal, altera de la misma forma a las reservas de agua como a los caudales punta, especialmente durante los meses de verano, ya que durante estos meses los caudales punta no se encuentran influenciados por las precipitaciones, sino que responden más bien a la existencia de reservas de agua $(24,25)$ En este sentido, Duncan (26), con información recolectada en Moutere, Nueva Zelandia, señala que la humedad del suelo podría decrecer alrededor de un $30 \%$, mientras las recargas podrían disminuir hasta cerca de un $70 \%$; sin embargo, este tipo de resultados son aplicables sólo a nivel local.

Estos antecedentes muestran lo interesante que es estudiar en Chile, un país con un importante sector forestal basado en las plantaciones de $P i$ nus radiata (D. Don), la influencia que estas masas boscosas ejercen sobre los distintos componentes del ciclo hidrológico.

El proceso de forestación masiva que se ha producido en Chile a partir de la década del 70 hasta la fecha, ha hecho surgir con fuerza la idea de que las plantaciones forestales reducen las disponibilidades de agua y, por ende, actúan disminuyendo la producción de agua en las cuencas hidrográficas. Según distintas experiencias, lo normal es pensar que la producción de agua se vea reducida, porque gran parte del uso del agua, está destinada a la transformación del paisaje. Es decir, una forestación masiva que reemplaza a una vegetación muy rala o se ubica en suelos descubiertos está generando una gran cantidad de biomasa. Por ello, es esperable que una forestación masiva demande una mayor cantidad de agua. (6, 8, 21, 22, 27, 28, 29, 30, 31, 32).

En este contexto, este artículo intenta obtener respuestas ligadas a la influencia de las plantaciones forestales en la producción de agua de la cuenca del río Purapel, la cual presenta características subhúmedas y se ubica en la zona central de Chile. Para ello, se analizaron variables hidrológicas, tanto a nivel mensual como anual, tratando de inferir el grado de influencia de la cobertura forestal en el escurrimiento superficial. La hipótesis sobre la cual se trabajó es que las plantaciones masivas inciden en una menor producción de agua. $\mathrm{Si}$ la hipótesis se comprobaba, el paso siguiente sería determinar en qué proporción se produce la disminución hídrica de la cuenca.

\section{MATERIAL Y METODOS}

La cuenca del río Purapel se localiza en el secano costero de la zona central de Chile y presenta una superficie de $264,6 \mathrm{~km}^{2}$ (figura 1). Sus cotas mínimas y máximas varían entre los 96 y 540 ms.n.m., respectivamente. La orientación del cauce principal va de oeste a suroriente, con una longitud del cauce principal de $25 \mathrm{~km}$. Posee un regimen hidrológico netamente pluvial, presentando una precipitación media anual, entre los años 1960 y 2000, de 812,4 mm. Esta cuenca presentó (según el análisis multitemporal de vegetación) al año 1997 una cobertura forestal sobre el 70\%, principalmente plantaciones de Pinus radiata (D. Don) y bosque nativo.

Los suelos de la cordillera de la costa, donde se ubica la cuenca del río Purapel, están constituidos principalmente por rocas plutónicas paleozoicas, granitoide rico en cuarzo y diorita cuarcífera (33). Los intensos procesos de meteorización que actuaron sobre estas rocas graníticas permitieron la formación in situ de un suelo residual de espesor variable, de baja fertilidad, permeabilidad y tasa de infiltración y propenso a la erosión, lo que contribuye a una alta escorrentía superficial concentrada en los meses lluviosos (de abril a octubre), lo cual decrece rápidamente después de los eventos de precipitación (34).

El estudio de la cobertura vegetal se realizó a través de un análisis multitemporal de la vegetación en la cuenca, que buscó establecer cambios vegetacionales y su influencia en la producción de agua. De esta forma, se determinó el tipo de cobertura vegetal en tres instantes, en el año 1955, 1978 y 1997. Para el primer año se utilizó cuatro mosaicos de CIREN (Centro de Información de Recursos Naturales), escala 1:20.000, confeccionado a partir de fotografías verticales del año 1955. Para 


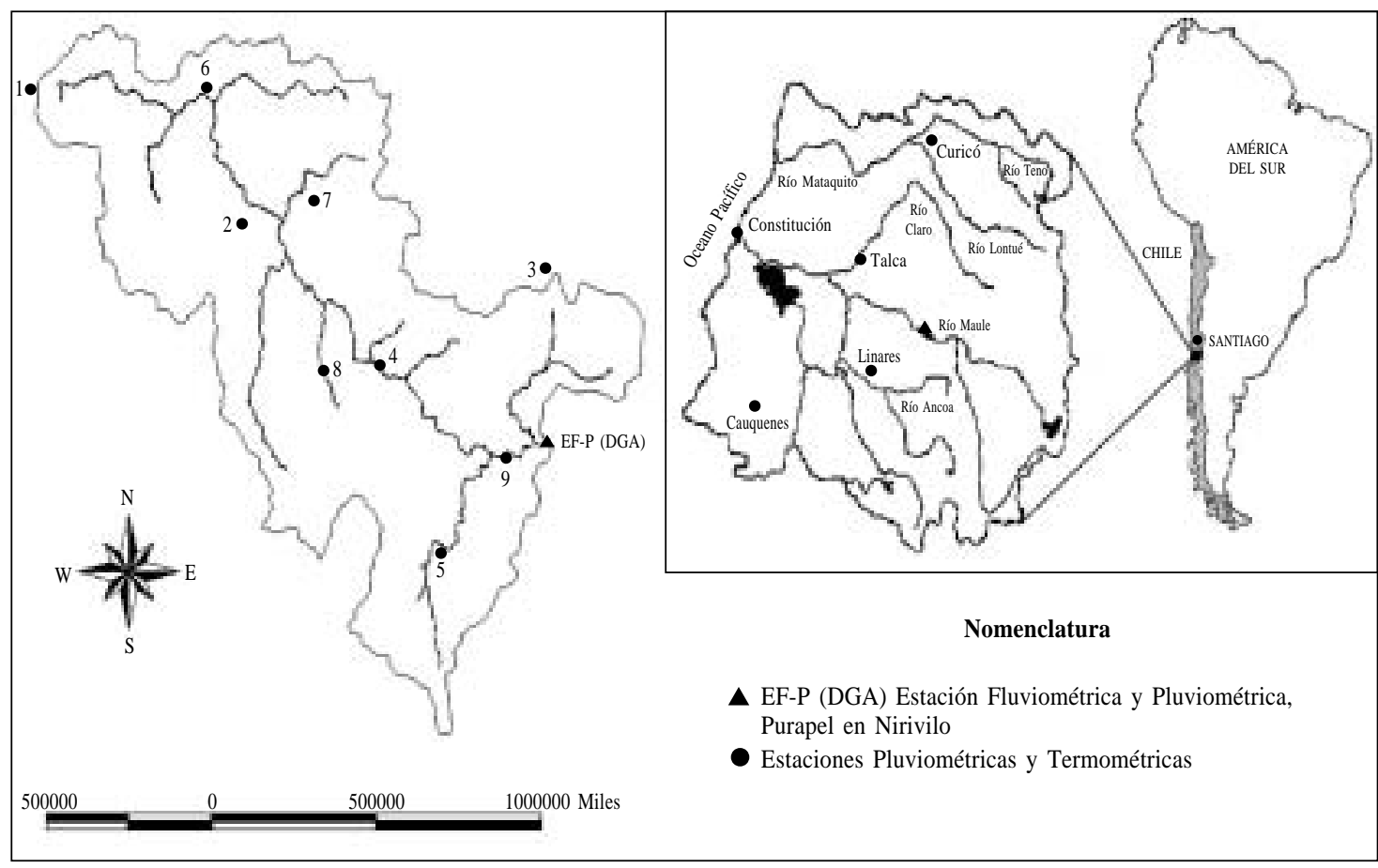

Figura 1. Plano de Ubicación Cuenca del Río Purapel. Map showing the location of Purapel river basin.

los años 1978 y 1997, se contó con ortofotos, confeccionadas a partir de fotografías aéreas. Luego, a través de fotointerpretación de la información, se determinó y clasificó la vegetación existente en cada año, información que se digitalizó mediante el software Arc/Info 3.5, pudiéndose elaborar cartografía digital mediante el software Arc view 3.1. De este análisis de la vegetación, se determinó que existian dos períodos de características distintas; el comprendido entre los años 1960-1978, que presentó mayor superficie cubierta por bosque nativo, mientras que entre los años 1979-2000, existe mayor superficie forestal cubierta por plantaciones de $P i$ nus radiata (D. Don). Estas diferencias vegetacionales se consideraron en los análisis estadísticos, considerando a cada intervalo temporal como períodos a contrastar y analizar.

La información pluviométrica y fluviométrica, entre los años 1960 y 2000, fue facilitada por la Dirección General de Aguas (DGA), dependiente del Ministerio de Obras Públicas de Chile. Además, se instalaron 9 pluviómetros en el año 2002, que permitieron mejorar la distribución espacial de las precipitaciones. De esta forma, se analizó el comportamiento de 3 variables del balance hídrico (precipitaciones, caudales y reservas), más dos variable auxiliares (coeficientes de escorrentía y caudales máximos).

Para las precipitaciones se utilizaron registros diarios de las estaciones Purapel en Nirivilo y de la estación Constitución, que tenían información entre los años 1992-2000. Con estas estaciones se estimaron las precipitaciones medias areales de la cuenca a nivel mensual y anual mediante los Polígonos de Thiessen $(4,35,36)$, dado que Pizarro et al, (36), lo señala como un método adecuado para la zona. Para los caudales, se utilizaron registros limnigráficos horarios entre 1960 y 2000, con los cuales, y utilizando las curvas de descarga, se construyeron los hidrogramas. Las reservas, a nivel mensual y anual, fueron determinadas integrando una función que modela el caudal recesivo (Q), de la forma [1]

$$
\mathrm{Q}=\mathrm{Q}_{0} * \mathrm{e}^{-\alpha(\Delta \mathrm{t})}
$$

donde, $\mathrm{Q}_{\mathrm{o}}$ : caudal inicial; e: coeficiente de Neper; $\alpha$ : coeficiente de agotamiento y $\Delta \mathrm{t}$ : diferencia de tiempo. Así, se determinaron las reservas iniciales y finales por mes. 
Los coeficientes de escorrentía (C), a nivel mensual y anual, se obtuvieron a partir del cociente entre la precipitación efectiva y la precipitación total caída sobre la cuenca. Así, la precipitación efectiva se calculó en base a lapsos horarios, separando el hidrograma total en el correspondiente al de aguas superficiales y el de aguas subterráneas (figura 2). Para los hidrogramas en que no fue posible determinar el punto de término de la escorrentía superficial y comienzo de la subterránea, éste se estimó mediante el método de regresión lineal. Para ello se estableció una correlación entre el punto de término del hidrograma, en horas, y el caudal máximo de la crecida $\left(\mathrm{m}^{3} / \mathrm{s}\right)$. A estas regresiones se les aplicaron algunos supuestos del método de mínimos cuadrados, como Normalidad, Homocedasticidad y no-autocorrelación, junto con probar la significancia de los parámetros. Así, las precipitaciones efectivas de un mes i y un año $\mathrm{j}$, se calcularon como el cociente entre el volumen de escorrentía directa para un mes i y un año $\mathrm{j}\left(V_{i j}\right)$ y el área de la cuenca $(A)$, expresión [2]:

$$
\mathrm{Pe}_{\mathrm{ij}}=\frac{\mathrm{V}_{\mathrm{ij}}}{\mathrm{A}}
$$

Entonces, y según Chow et al. (37), si $\left(P_{i j}\right)$ es la precipitación en un mes i y un año j, y $\left(P e_{i j}\right)$ precipitación efectiva, el coeficiente de escorrentía $\left(C_{i j}\right)$ para un mes i, en un año j, puede definirse como [3]:

$$
C_{i j}=\frac{P e_{i j}}{P_{i j}}
$$

Se utilizó esta variable porque el coeficiente de escorrentía es muy sensible frente a cambios en el comportamiento hidrológico superficial de la cuenca $(17,37)$.

Los caudales máximos (Qp) se seleccionaron tanto a nivel mensual como anual desde la serie 1960-2000, clasificando los caudales en estivales (caudales de noviembre a marzo) e invernales (caudales de abril a octubre). Esta clasificación se realizó con el fin de mejorar los análisis estadísticos posteriores, analizando principalmente los caudales estivales, ya que durante este período no existe influencia de precipitaciones, lo que permite determinar si se ha producido una variación de los caudales a través del tiempo y si esto se debe a las plantaciones forestales realizadas en la cuenca entre 1960 y 2000.

Para los análisis estadísticos de la información, se comprobó previamente que las series de datos no cumplían los supuestos básicos de normalidad de los residuos e igualdad de las varian-

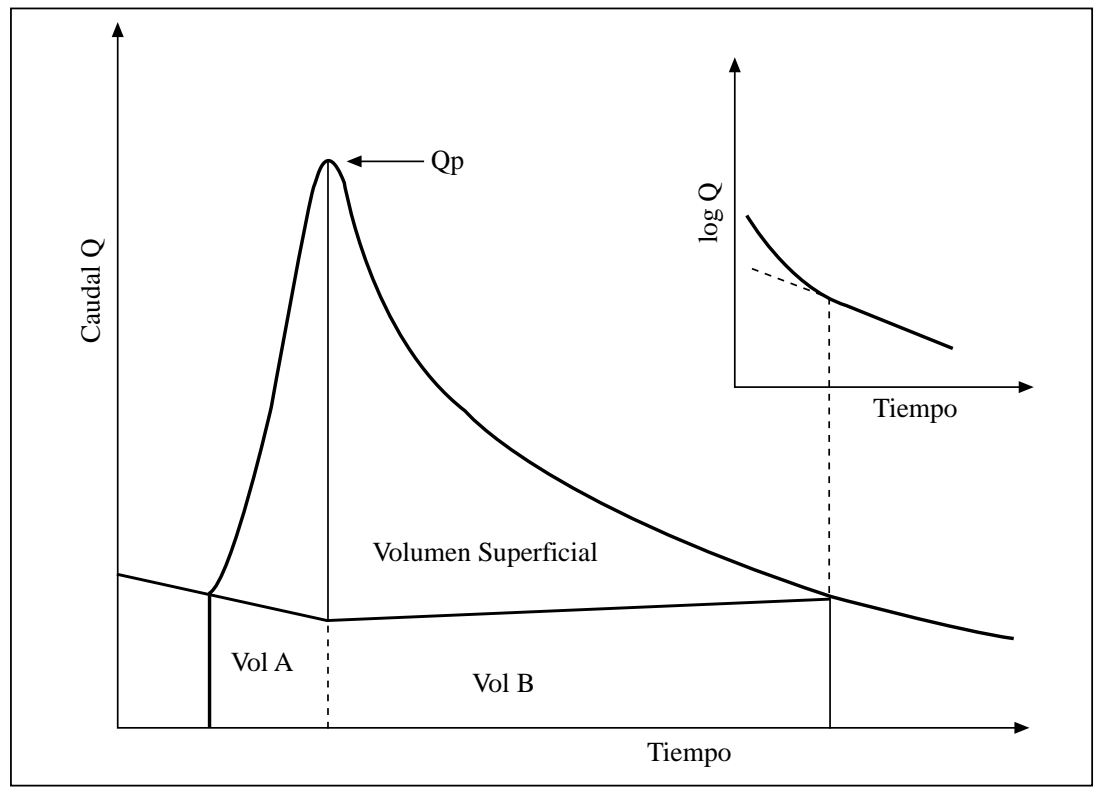

Figura 2. Forma típica de un hidrograma de crecida y sus características (Chow et al., 1994).

Discharge hydrograph and its components 
zas, descartando el uso del análisis de varianza (ANDEVA), optándose por utilizar pruebas no paramétricas. Así, primeramente se utilizó la prueba de Kruskal-Wallis y, si esta prueba indicaba que existían variaciones, posteriormente se aplicaba la prueba U de Mann-Whitney, que señalaba si existían diferencias significativas entre dos grupos de datos. Estas pruebas se aplicaron a nivel mensual, anual, por décadas y entre períodos. Paralelamente, se realizaron análisis gráficos mediante promedios móviles, calculados en intervalos de tres años para el total de la serie estudiada (40 años), los cuales permitieron suavizar la información, cancelando el efecto de la variación aleatoria (34).

\section{RESULTADOS Y DISCUSION}

Las variaciones en la composición vegetacional producidas en la cuenca del río Purapel durante las últimas décadas han sido masivas, produciéndose cambios en el tipo de vegetación desde bosques nativos, matorrales y terrenos agrícolas, a extensas áreas de plantaciones forestales. Se puede señalar que la principal variación vegetacional de la cuenca, obedece al cambio de un bosque nativo, con una gran variedad de especies, hacia plantaciones de Pinus radiata (D. Don). En este proceso de modificación de la cubierta vegetal, se considera el año 1978 como un punto de quiebre, ya que a partir de ese momento se produ- ce un incremento de las plantaciones forestales presentes en la cuenca (cuadro 1, figura 3), a partir de donde se puede inferir el importante cambio en la cobertura vegetal. Este cambio está relacionado con una remoción importante de bosque nativo, principalmente de los tipos esclerófilo y roble-hualo, desde un $63,2 \%$ de la superficie en el año 1955 , hasta un $19,2 \%$ en el año 1997 , para dar paso a plantaciones forestales casi exclusivamente con la especie Pinus radiata (D. Don), que desde una nula presencia en 1955, llega a ocupar el 51,7\% de la superficie al año 1997.

Los análisis de precipitaciones se iniciaron examinando los valores puntuales de precipitación y se observó que existían valores mayores en la década del 80, lo que hizo suponer que esta década había sido más lluviosa. Para poder corroborar esto, se sumó el total de lluvia caída en la cuenca durante los 40 años, obteniéndose un valor de $32.495,3 \mathrm{~mm}$. Los valores totales por décadas se muestran en el cuadro 2, donde se observa que efectivamente en los 80 ocurrieron montos superiores de precipitación que los ocurridos en las décadas del 60, 70 y 90. Además, en la década del 80 es cuando ocurre la mayor cantidad de años con precipitaciones anuales que superan los mil milímetros, cinco años, registrándose además el mayor monto de toda la serie estudiada, año hidrológico 82/83 con 1.417,2 $\mathrm{mm}$. De lo anterior, se puede asumir que las precipitaciones se vieron incrementadas en sus montos en la década del 80 respecto de las demás décadas.

\section{CUADRO 1}

Evolución de la superficie forestal, cuenca río Purapel.

Land use evolution in Purapel river basin.

\begin{tabular}{|l|r|r|r|r|r|r|}
\hline & \multicolumn{7}{|c|}{ Superficie (ha) } \\
\hline Clasificación & Año 1955 & \multicolumn{1}{|c|}{$\%$} & \multicolumn{1}{|c|}{ Año 1978} & \multicolumn{1}{c|}{ Año 1997} & $\%$ \\
\hline Bosque de P. radiata (D. Don) & 0,0 & 0,0 & $5.115,5$ & 19,3 & $13.677,9$ & 51,7 \\
Bosque Nativo & $16.737,9$ & 63,2 & $13.698,8$ & 51,8 & $5.214,6$ & 19,7 \\
Poblados & 16,8 & 0,1 & 17,5 & 0,1 & 34,4 & 0,1 \\
Terrenos de Uso Agrícola & $1.464,9$ & 5,5 & $1.092,3$ & 4,1 & $2.393,4$ & 9,0 \\
Praderas & $2.486,4$ & 9,4 & $1.926,3$ & 7,3 & 984,7 & 3,7 \\
Matorral Abierto & $2.885,3$ & 10,9 & $1.708,9$ & 6,5 & 942,3 & 3,6 \\
Matorral Semi-denso & $2.557,9$ & 9,7 & $2.081,0$ & 7,9 & $2.172,2$ & 8,2 \\
Matorral Denso & 317,3 & 1,2 & 826,2 & 3,1 & $1.047,0$ & 4,0 \\
\hline TOTAL & $26.466,5$ & 100 & $26.466,5$ & 100 & $26.466,5$ & 100 \\
\hline
\end{tabular}




\section{CUADRO 2}

Precipitación total caída por década.

Total rainfall by decade.

\begin{tabular}{|c|c|c|c|c|}
\hline Década & $\begin{array}{c}\text { Precipitación) } \\
\text { Total }(\mathrm{mm})\end{array}$ & $\%$ & Promedio $(\mathrm{mm})$ & $\begin{array}{c}\text { Año más lluvioso } \\
\text { y valor asociado }\end{array}$ \\
\hline 60 & $6.606,8$ & 20,3 & 660,7 & $1963(1.133,8)$ \\
70 & $8.554,7$ & 26,3 & 855,5 & $1972(1.393,6)$ \\
80 & $9.703,2$ & 30 & 970,3 & $1982(1.417,2)$ \\
90 & $7.630,6$ & 23,4 & 763,1 & $1992(1.225,7)$ \\
\hline Total & $32.495,3$ & 100 & 812,4 & \\
\hline
\end{tabular}

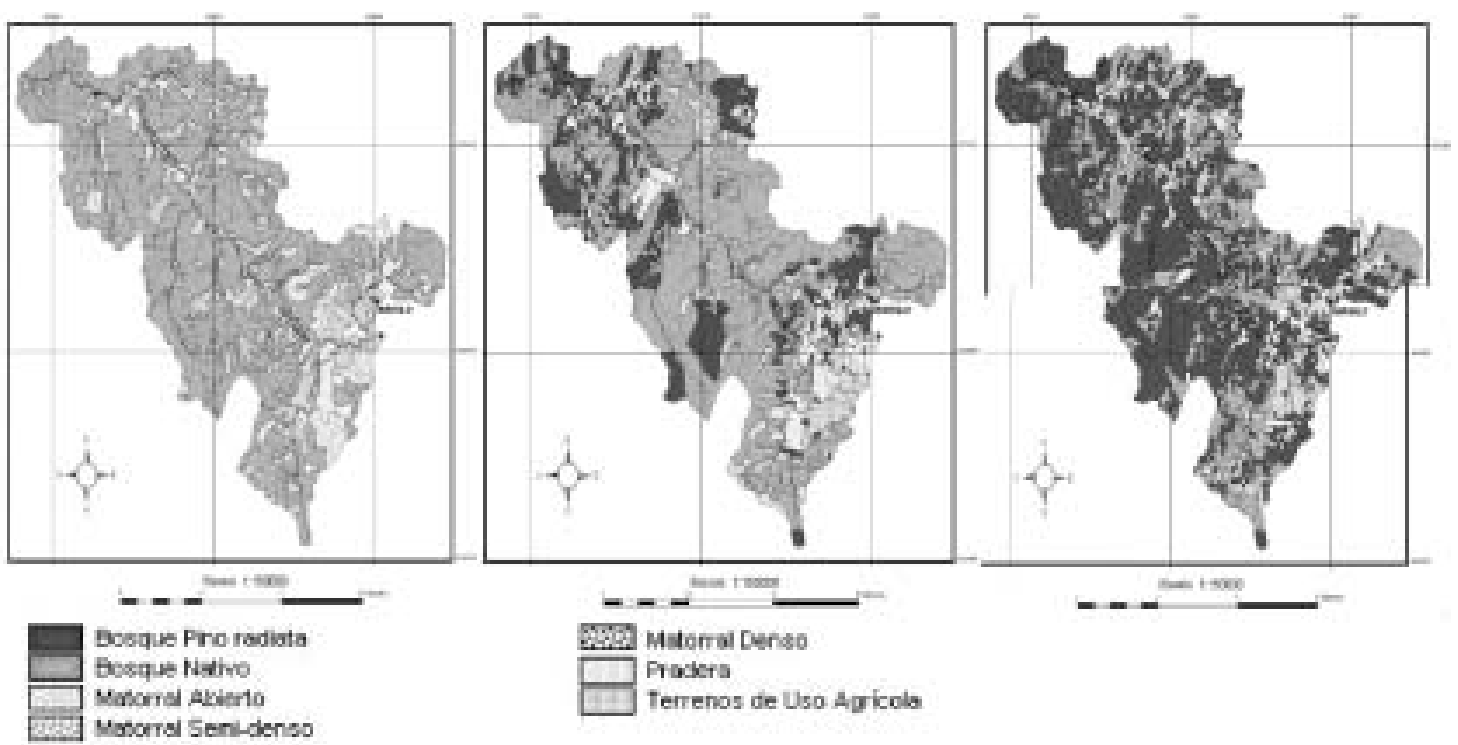

Figura 3. Variación de la ocupación del suelo en relación al tipo de cubierta vegetal en los años 1957, 1978 y 1997. Variation of land use in 1957, 1978 and 1997.

Las precipitaciones areales, mensuales y anuales de la cuenca, estimadas según los polígonos de Thiessen, fueron corregidas con información de los 9 pluviómetros instalados en el área de estudio durante el año 2002. Con estos registros se pudo obtener un factor de corrección para cada mes (38). Estos factores de corrección presentaron valores muy cercanos a 1 , que no cambiaron significativamente los valores estimados por los polígonos.

La prueba U de Mann-Whitney, que compara el periodo 1961/1978 con el periodo 1979/2000 para meses húmedos y secos por año, muestra que el periodo 1961/1978 no presenta diferencias significativas con el periodo 1979/2000 a nivel de la serie total de meses. La misma prueba aplicada a nivel de datos anuales reveló que sí existen diferencias significativas para las precipitaciones entre ambos periodos (cuadro 3).

Cuando se hizo el análisis considerando sólo la suma de las precipitaciones de los meses húmedos y los secos, no se detectaron en ambos casos diferencias significativas según la prueba $\mathrm{U}$ de Mann-Whitney (cuadro 4).

Los caudales medios mensuales comparados entre décadas mostraron que existen variaciones entre los valores de la década del 80 al contrastar- 


\section{CUADRO 3}

Prueba U de Mann-Whitney de comparación de precipitaciones entre periodos 1961/1978 v/s 1979/2000.

U de Mann-Whitney test for rainfall comparison between 1961/1978 and 1979/2000 periods.

\begin{tabular}{|l|c|c|c|}
\hline Comparación & $\mathrm{Zc}$ & $\mathrm{Zt}^{*}$ & Conclusión* \\
\hline Total de meses & 0,34 & $\pm 1,96$ & No existen diferencias significativas \\
Todos los años & $-1,99$ & $\pm 1,96$ & Existen diferencias significativas \\
\hline
\end{tabular}

* Considerando un nivel de significancia estadística de 0,05 .

\section{CUADRO 4}

Prueba U de Mann-Whitney de comparación de precipitaciones para meses húmedos y secos entre periodos 1961/1978 v/s 1979/2000.

U de Mann-Whitney test for rainfall comparison in wet and dry months, between1961/1978 and 1979/2000 periods.

\begin{tabular}{|l|c|c|c|}
\hline Comparación & $\mathrm{Zc}$ & $\mathrm{Zt}^{*}$ & Conclusión \\
\hline Meses húmedos & 1,10 & $\pm 1,96$ & No existen diferencias significativas \\
Meses secos & 0,52 & $\pm 1,96$ & No existen diferencias significativas \\
\hline
\end{tabular}

* Considerando un nivel de significancia estadística de 0,05 .

la con las demás décadas (cuadro 5). Esta diferencia se puede explicar por los mayores valores de precipitación e intensidad ocurridos en los 80 . Asimismo, si se compara el periodo 1961/1978 con el periodo 1979/2000, se aprecia que no existen diferencias significativas entre ambos periodos, y ello a nivel anual y mensual, lo cual estaría dando indicios que el cambio del tipo de cubierta vegetal no estaría afectando la producción de agua de la cuenca (cuadro 6).

\section{CUADRO 5}

Prueba U de Mann-Whitney de comparación de caudales entre décadas

U de Mann-Whitney test for runoff comparison, between decades

\begin{tabular}{|l|c|c|c|c|c|c|}
\cline { 2 - 7 } \multicolumn{1}{c|}{} & \multicolumn{6}{c|}{ Comparación de Décadas } \\
\hline Variable & $60-70$ & $60-80$ & $60-90$ & $70-80$ & $70-90$ & $80-90$ \\
\hline Caudal medio mensual & - & $* *$ & - & $* *$ & - & $* *$ \\
\hline
\end{tabular}

-: No existen diferencias entre décadas, considerando un nivel de significancia estadística de 0,05 .

**: Existen diferencias entre décadas, considerando un nivel de significancia estadística de 0,05 . 


\section{CUADRO 6}

Prueba U de Mann-Whitney de comparación de caudales entre periodos 1961/1978 v/s 1979/2000.

U de Mann-Whitney test runoff comparison between 1961/1978 and 1979/2000 periods.

\begin{tabular}{|l|c|c|c|}
\hline Comparación & $\mathrm{Zc}$ & $\mathrm{Zt} *$ & Conclusión \\
\hline Total de meses & $-0,98$ & $\pm 1,96$ & No existen diferencias significativas \\
Nivel anual & 1,39 & $\pm 1,96$ & No existen diferencias significativas \\
\hline
\end{tabular}

* Considerando un nivel de significancia estadística de 0,05 .

Por otra parte, si se realiza un análisis gráfico de la variable precipitación, se puede observar el incremento de las precipitaciones ocurridas en la década del 80 (figura 4), apreciándose los montos anuales que superan la media de precipitaciones para el período $812,4 \mathrm{~mm}$. Así, se observó que 5 años de la década de los 80 superan la media, condición que no ocurre en ninguna otra década. Asimismo, si se realiza un gráfico conjunto de las variables precipitación y caudal, se advierte la importante dependencia de los caudales respecto a las precipitaciones y de cómo los valores se incrementan durante los años ochenta (figura 5).

Las variaciones de reservas presentaron un comportamiento estadísticamente distinto respecto a las otras variables. Así, las reservas a nivel anual y por décadas presentaron variaciones significativas según la prueba Kruskall-Wallis (cua-

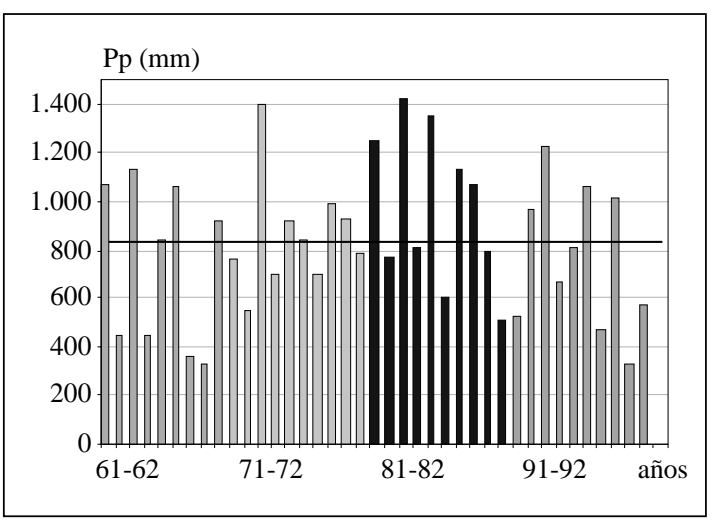

Figura 4. Variación de los valores anuales de precipitación, 1960-2000.

Variation of yearly rainfall values, 1960/2000. dro 7). Esta diferencia se presentó al comparar la década del 80 respecto a las otras décadas, según la prueba U de Mann-Whitney (cuadro 8).

\section{CUADRO 7}

Prueba de Kruskal-Wallis de comparación de reservas entre décadas.

Kruskal-Wallis test for storage comparison.

\begin{tabular}{|l|l|l|c|}
\hline Variable & Estadístico & Valor $\mathrm{p}$ & Conclusión* \\
\hline Dec60 & & & \\
Dec70 & 18,3954 & 0,000364 & Existen variaciones \\
Dec80 & & & \\
Dec90 & & & \\
\hline
\end{tabular}

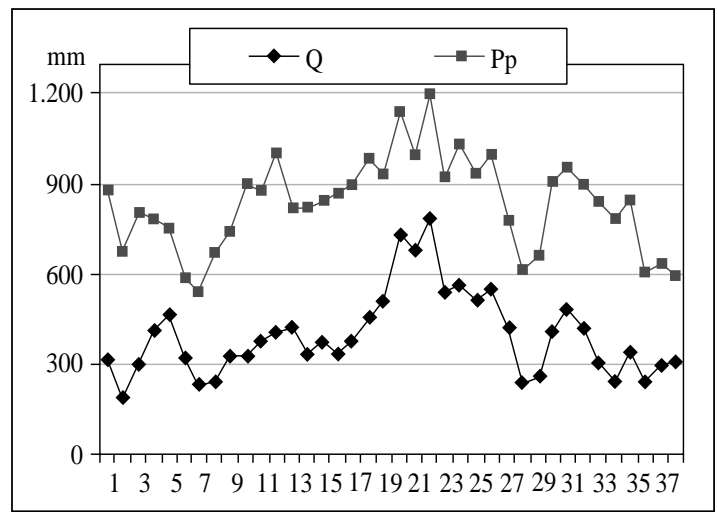

Figura 5. Variación de los promedios móviles de precipitaciones y el caudal, 1960-2000.

Variation of moving average for rainfall and runoff. 


\section{CUADRO 8}

Prueba U de Mann-Whitney de comparación de reservas entre décadas.

U de Mann-Whitney test for storage comparison between decades.

\begin{tabular}{|l|c|c|c|c|c|c|}
\cline { 2 - 7 } \multicolumn{1}{c|}{} & \multicolumn{6}{c|}{ Comparación de Décadas } \\
\hline Variable & $60-70$ & $60-80$ & $60-90$ & $70-80$ & $70-90$ & $80-90$ \\
\hline Reservas anuales & - & $* *$ & - & $* *$ & - & - \\
\hline
\end{tabular}

-: No existen diferencias entre décadas, considerando un nivel de significancia estadística de 0,05 .

**: Existen diferencias entre décadas, considerando un nivel de significancia estadística de 0,05 .

Al contrastar los periodos 1961/1978 con 1979/ 2000 se aprecia que según la prueba U de MannWhitney sí existen diferencias significativas a nivel mensual, pero no a nivel anual (cuadro 9). Asimismo y contrariamente a lo esperado, es en el segundo periodo en que estos valores se hacen mayores (figura 6), lo que obedecería a las mayores precipitaciones de los 80 , que a cambios de la cobertura vegetal; o a que el bosque de Pinus radiata (D. Don) genera mayores reservas de aguas que el bosque nativo.

Los valores promedio del coeficiente de escorrentía (C) mensual se presentan en el cuadro 10, destacando los niveles cercanos a 0 de los meses de estiaje, y los valores de los meses invernales en que no se supera el valor $\mathrm{C}=0,12$. Además, se observa que en la mayoría de los meses (9) los valores no superan el $10 \%$ de coeficiente de escorrentía promedio.

El valor del $\mathrm{C}$ anual como promedio por décadas no presenta mayores variaciones para las dé-

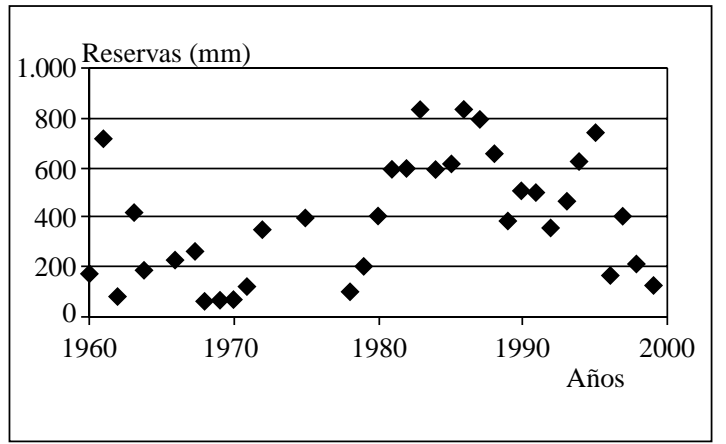

Figura 6. Valores anuales de la variación de reservas. Yearly values of storage variation.

cadas 60,70 y 90, presentando un mayor valor en la década del 80 (cuadro 11). Se esperó que la década del 90, por las mayores superficies de plantaciones forestales, presentase valores menores del $\mathrm{C}$ en comparación con las décadas del 60 y 70 . Sin embargo, ello no fue así, y muy por el contra-

\section{CUADRO 9}

Prueba U de Mann-Whitney de comparación de reservas entre períodos 1961/1978 v/s 1979/2000.

U de Mann-Whitney test for storage comparison between $1961 / 1978$ and 1979/2000 periods.

\begin{tabular}{|l|c|c|c|}
\hline Comparación & $\mathrm{Zc}$ & $\mathrm{Zt}^{*}$ & Conclusión* \\
\hline Total de meses & 7,26 & $\pm 1,96$ & Existen diferencias significativas \\
Nivel anual & 2,79 & $\pm 1,96$ & No existen diferencias significativas \\
\hline
\end{tabular}

* Considerando un nivel de significancia estadística de 0,05 


\section{CUADRO 10}

Valores promedios de C para cada mes entre 1960-2000.

Mean values of monthly runoff coefficient, 1960-2000.

\begin{tabular}{|c|c|c|c|c|c|c|c|c|c|c|c|c|}
\cline { 2 - 13 } \multicolumn{1}{c|}{} & \multicolumn{10}{c|}{ Meses } \\
\cline { 2 - 12 } & Abr & May & Jun & Jul & Ago & Sep & Oct & Nov & Dic & Ene & Feb & Mar \\
\hline Valores & 0,0087 & 0,038 & 0,113 & 0,117 & 0,102 & 0,076 & 0,036 & 0,018 & 0,016 & 0,002 & 0,000 & 0,008 \\
\hline
\end{tabular}

rio, el valor promedio del coeficiente de escorrentía es superior en los 90 que en los 60 y 70. Esto se debería a los montos de precipitaciones y a su ocurrencia temporal, porque la precipitación es una variable de influencia directa, mientras que el tipo de cobertura vegetal y las condiciones de suelo influyen indirectamente a través del cálculo de las precipitaciones efectivas.

Por otra parte, se compararon los datos por década a nivel anual. El cuadro 12 muestra los resultados de la prueba U de Mann-Whitney, en que se comprueba que la década del 80 es la que presenta diferencias significativas con las restantes, en tanto que las del 60,70 y 90 no presentaron diferencias entre ellas.

Al comparar entre los periodos 1961/1978 con 1979/2000 se observa que según la prueba U de Mann-Whitney no existen diferencias significativas a nivel anual (cuadro 13), con lo cual no existen antecedentes para determinar que se ha producido una variación en los coeficientes de escorrentía entre los dos periodos analizados.

Los caudales máximos (Qp), aparte de ser comparados entre décadas y períodos, se compararon

\section{CUADRO 11}

Valores promedio de $\mathrm{C}$ anuales por décadas entre 1960-2000.

Mean values of yearly runoff coefficient by decades between 1960 and 2000 .

\begin{tabular}{|c|c|}
\hline Década & Coeficiente de escorrentía promedio \\
\hline 60 & 0,050 \\
70 & 0,064 \\
80 & 0,102 \\
90 & 0,070 \\
\hline
\end{tabular}

entre valores invernales y estivales. Como era de esperar, los mayores valores ocurren durante los meses de invierno (mayo, junio, julio y agosto). Por otra parte, si se analiza cuándo ocurren los mínimos caudales de los valores estivales, éstos se presentan en los meses de enero y marzo de la década del 90, mientras que sus valores más altos tienden a ocurrir en la década del 80 (noviembre, diciembre y enero).

Al comparar los caudales máximos promedio anual, con el promedio de los 40 años $\left(21,65 \mathrm{~m}^{3} / \mathrm{s}\right)$,

\section{CUADRO 12}

Prueba U de Mann-Whitney de comparación de C entre décadas.

U Mann-Whitney test for runoff coefficient comparison between decades

\begin{tabular}{|l|c|c|c|c|c|c|}
\cline { 2 - 7 } \multicolumn{1}{c|}{} & \multicolumn{6}{c|}{ Comparación de Décadas } \\
\hline Variable & $60-70$ & $60-80$ & $60-90$ & $70-80$ & $70-90$ & $80-90$ \\
\hline Coeficiente de escorrentía anual & - & $* *$ & - & $* *$ & - & $* *$ \\
\hline
\end{tabular}

-: No existen diferencias entre décadas, considerando un nivel de significancia estadística de 0,05 .

**: Existen diferencias entre décadas, considerando un nivel de significancia estadística de 0,05 . 


\section{CUADRO 13}

Prueba U de Mann-Whitney de comparación de C entre periodos 1961/1978 v/s 1979/2000.

U Mann-Whitney test for runoff coefficient comparison between $1961 / 1978$ and $1979 / 2000$ periods.

\begin{tabular}{|l|c|c|c|}
\hline Comparación al nivel anual & $\mathrm{Zc}$ & $\mathrm{Zt}^{*}$ & Conclusión \\
\hline Coeficiente de escorrentía anual & 1,58 & $\pm 1,96$ & $\begin{array}{c}\text { No existen diferencias } \\
\text { significativas }\end{array}$ \\
\hline
\end{tabular}

* Considerando un nivel de significancia estadística de 0,05.

la cantidad de datos sobre y debajo este promedio resultó ser similar, con 19 valores sobre el caudal máximo promedio anual y 21 datos por debajo de esta cifra. Sin embargo, durante los primeros 20 años en estudio, la mayor cantidad de datos se ubica bajo el promedio anual, mientras que a partir de la segunda mitad, la mayoría de los caudales máximos anuales se ubican por sobre el valor promedio (figura 7). Esto refleja un incremento importante de los caudales máximos anuales a partir de la década del 80 , presentándose el mayor valor de caudal máximo anual en el año 91/92, con 54,5 m³.

Al aplicar la prueba U de Mann-Whitney a los caudales máximos promedio anuales, se observa que sólo existen diferencias para la comparación hecha entre las décadas 60-80 y 70-80 (cuadro 14). Al realizar la misma prueba, para los caudales máximos anuales, se observa que no existen diferencias significativas en ninguna de las comparaciones, lo cual indica que los caudales máxi-

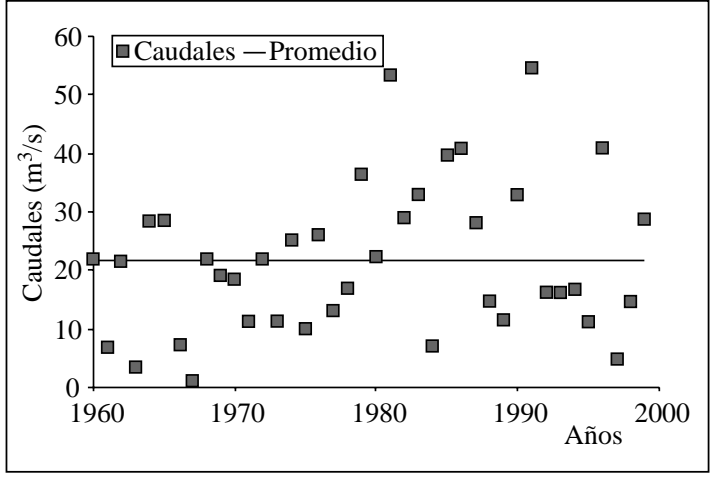

Figura 7. Caudales máximos promedio anual v/s promedio del periodo.

Mean yearly peak flows v/s period average.

mos de cada año se han mantenido similares a lo largo de los 40 años. Lo mismo ocurre en el caso de los caudales máximos estivales, los que tampoco presentaron diferencias significativas.

\section{CUADRO 14}

Prueba U de Mann- Whitney de comparación de caudales máximos entre décadas.

U Mann-Whitney test for peak flows comparison between decades.

\begin{tabular}{|l|c|c|c|c|c|c|}
\cline { 2 - 7 } \multicolumn{1}{c|}{} & \multicolumn{6}{c|}{ Comparación de Décadas } \\
\hline Variable & $60-70$ & $60-80$ & $60-90$ & $70-80$ & $70-90$ & $80-90$ \\
\hline $\begin{array}{l}\text { Caudales máximos anuales } \\
\text { Caudales máximos estivales }\end{array}$ & - & - & - & - & - & - \\
$\begin{array}{l}\text { Promedio anual de caudales } \\
\text { máximos }\end{array}$ & - & $* *$ & - & $* *$ & - & - \\
\hline
\end{tabular}

-: No existen diferencias entre décadas, considerando un nivel de significancia estadística de 0,05 . **: Existen diferencias entre décadas, considerando un nivel de significancia estadística de 0,05. 
El hecho de que para los caudales máximos estivales no existan diferencias entre décadas, constituye un dato importante, ya que los caudales estivales no se ven influenciados por las precipitaciones sino más bien por las reservas de agua, y éstas a su vez por la mayor o menor presencia de vegetación; por lo tanto, cambios importantes de cobertura vegetal, deberían reflejar cambios de caudal estival, lo cual no se advierte en la cuenca del río Purapel.

Al comparar entre los períodos 1960/1978 y 1979/2000, los resultados muestran que no existen diferencias significativas para los promedios anuales los caudales máximos anuales y para los caudales máximos estivales (cuadro 15). Esto reafirma lo ya señalado, respecto a las diferencias que presenta la década del 80 respecto de las demás décadas.
Finalmente, al realizar un análisis gráfico de todas las variables por medio de los promedios móviles, se observó un comportamiento similar de las tendencias, estando marcadas por la conducta que establecen las precipitaciones (figura 8). La serie muestra un marcado incremento en los años 80 en comparación con el resto del período (figura 9). $\mathrm{Si}$ a estos resultados se suman los obtenidos en las pruebas estadísticas donde todas las variables presentaron valores más altos en su análisis cuando se estudiaba la década del 80 y su contraste con las demás décadas, entonces se puede decir que es esta década la que presenta condiciones hidrológicas distintas. La explicación a esta situación se encontraría en las precipitaciones ocurridas durante esta década, las cuales son mayores en montos e intensidades comparada con las demás décadas, siendo estas características las

\section{CUADRO 15}

Prueba U de Mann-Whitney de comparación de caudales máximos entre periodos 1961/1978 v/s 1979/2000.

U Mann-Whitney test for peak flows comparison between 1961/1978 and $1979 / 2000$ periods.

\begin{tabular}{|l|c|c|c|}
\hline Comparación al nivel anual & \multicolumn{1}{|c|}{$\mathrm{Zc}$} & $\mathrm{Zt}^{*}$ & Conclusión \\
\hline Máximo caudal punta anual & $-1,69$ & $\pm 1,96$ & No existen diferencias significativas \\
Máximo caudal punta estival & $-1,58$ & $\pm 1,96$ & No existen diferencias significativas \\
Caudal punta promedio anual & $-1,91$ & $\pm 1,96$ & No existen diferencias significativas \\
\hline
\end{tabular}

* Considerando un nivel de significancia estadística de 0,05.

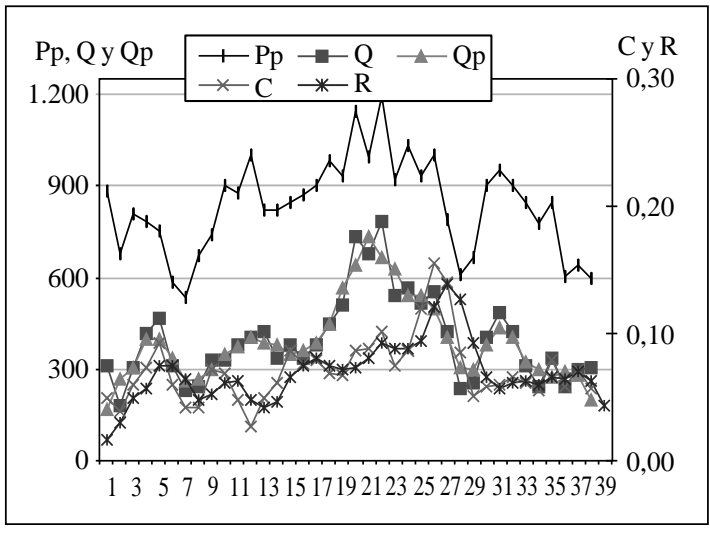

Figura 8. Variación de los promedios móviles anuales, 1960-2000.

Variation of yearly moving average, 1960/2000.

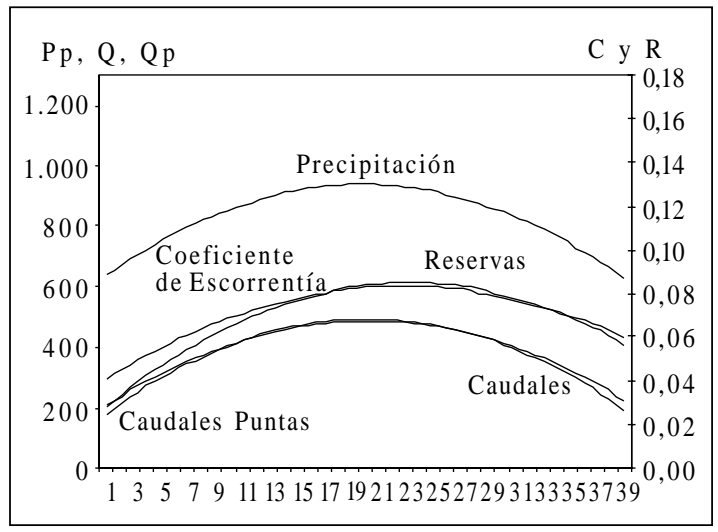

Figura 9. Gráfica de tendencias para la serie, 19602000.

Trends graph for 1960-2000 values. 
responsables del incremento en los valores de las variables analizadas. Según estos antecedentes, la precipitación es la variable más influyente sobre los distintos componentes del balance hídrico, lo cual explica por qué los dos tipos de bosques analizados, nativos y plantaciones forestales de Pinus radiata (D. Don), presentan un comportamiento hidrológico similar. Entonces, es posible señalar que los valores de las variables hidrológicas estudiadas no estarían en función de las plantaciones de Pinus radiata (D. Don), sino que se encontrarían relacionadas con las precipitaciones y las intensidades con las que éstas caen sobre la cuenca.

\section{CONCLUSIONES}

En la cuenca del río Purapel, y según se desprende del análisis multitemporal de la vegetación realizado, se produjo un proceso masivo de sustitución, reemplazándose superficie de bosque nativo por plantaciones de Pinus radiata (D. Don), proceso que fue más notorio desde la segunda mitad de la década del 70 hasta la primera mitad de la década del 80, donde las plantaciones de pino insigne pasaron desde un $19,3 \%$ al $51,7 \%$ de la superficie, en desmedro de la superficie cubierta por bosque nativo que pasó de un 51,8\% a un $19,7 \%$.

El comportamiento hidrológico de los dos tipos de bosques en la cuenca del río Purapel se puede considerar en términos generales que es similar para el periodo 1961/1978 (de mayor superficie con bosque nativo) y el periodo 1979/ 2000 (con mayor influencia de plantaciones forestales) y ello en función de la inexistencia de diferencias significativas a nivel mensual y anual de las variables precipitación, caudal, coeficiente de escorrentía y caudales máximos. Sólo las reservas presentan un comportamiento distinto, ya que se incrementan en el período 1979/2000, cuando la superficie de la cuenca tiene una mayor influencia de plantaciones con "pino insigne". Los resultados obtenidos se ven ratificados al utilizar los valores del método del Número de Curva del U.S. Soil Conservation Service, donde se aprecia que los datos para los dos tipos de bosques analizados, son similares entre sí, lo cual corroboraría un similar comportamiento hidrológico de ambas masas forestales, por una parte, y desde otra óptica, determinaría que las aproximaciones en cifras del método del Número de Curva parecen ser las adecuadas.

El análisis de los resultados lleva a establecer que en la cuenca del río Purapel el elemento más determinante en el comportamiento hidrológico es la cantidad e intensidad de las precipitaciones caídas a nivel mensual y anual, y no el tipo de cobertura vegetal boscosa, en el contexto de la escala utilizada, $250 \mathrm{~km}^{2}$, existiendo una alta probabilidad de encontrar cambios en los valores totales escurridos si se utiliza una escala de trabajo menor.

Los registros de precipitación muestran que en la década de los años 80 se incrementaron de manera importante los montos y las intensidades ocurridas, elemento que a su vez ejerce un marcado control sobre el resto de las variables analizadas, según se pueden apreciar en las figuras 5,8 y 9 .

Los análisis hechos por otros autores indican que la influencia de la cubierta vegetal está en la modificación de las condiciones físico-químicas del perfil de suelo, afectando las condiciones de permeabilidad y la capacidad de retención de humedad, características que ocurren en la cuenca del río Purapel, pero que no son disímiles entre la cubierta vegetal nativa y la de plantaciones forestales, con lo cual no existen antecedentes para aseverar que un cambio de la cobertura vegetal boscosa haya provocado un cambio en el régimen hídrico.

\section{AGRADECIMIENTOS}

Los autores agradecen al Fondo Nacional de Desarrollo Científico y Tecnológico, quien hizo posible la realización de este estudio, como parte de un análisis mayor para la cuenca del río Purapel (Proyecto FONDECYT 1010590).

\section{BIBLIOGRAFIA}

(1) HUBER, A., y R. TRECAMAN. Efecto de la variabilidad interanual de las precipitaciones sobre el desarrollo de plantaciones de Pinus radiata (D. Don) en la zona de los arenales VIII Región, Chile. Bosque, 2002, vol. 23, $\mathrm{N}^{\circ} 2$, p. 43-49.

(2) LINDHOLM, P. \& I. STENBECK. Rainfall, soil loss and the effect of vegetation cover; Results of measurements in a tree plantation area in West Pokot District of Kenya. International Rural Development Center, Uni- 
versity of Agricultural Sciences, Working Paper $N^{\circ} 244$ Uppsala, Suecia, 1993, 25 p.

(3) LOPEZ. Restauración hidrológico forestal de cuencas y control de la erosión. Tragsa-Tragsatec, Ediciones Mundiprensa, Madrid, España, 1994, 898 p.

(4) LINSLEY, R., M. KOHLER, \& J. PAULUS. Hidrología para Ingeniero. Editorial Mc Graw-Hill, Bogotá, Colombia, 1977, $386 \mathrm{p}$.

(5) LAVABRE, J., D. SEMPERE-TORRES, \& F. CERNESSON. Etude du comportement hydrologique d'un petit bassin versant mediterraneen apres la destruction de l' ecosysteme forestier par un incendie. Journal de Hydrologique Continentale, vol. 6, $\mathrm{N}^{\circ}$ 2, 1991, p. 121-132.

(6) CALDER, I. Hydrologic effects of land-use change. Handbook of Hydrology, Editor D. Maidment. Mc GrawHill, Estados Unidos, 1992, 1.424 p.

(7) FARMER, D., M. SIVAPALAN \& C. JOTHITYANGKOON. Climate, soil, and vegetation controls upon the variability of water balance in temperate and semiarid landscapes: Downward approach to water balance analysis. Water Resources Research, 2003, vol. 39, $\mathrm{N}^{\circ}$ 2, p. 1-21.

(8) CALDER, I. Water use by forest, limits and controls. Tree Physiology, $\mathrm{N}^{\circ} 18,1998$, p. 625-631.

(9) FELLER, M. Water balance in Eucaliptus regnans, Eucaliptus oblicua and Pinus radiata forest in Victoria. Australian Forestry, 1981, vol. 44, No 3, p. 153-161.

(10) HUBER, A. y R. TRECAMAN. Efecto de la forestación de suelos arcillosos sobre el recurso hídrico en la zona de Collipulli, IX Región, Chile. Terra Australis, 2001, vol. 45, p. 49-60.

(11) PUTUHENA, W. \& I. CORDERY. Some hydrological effects of changing forest cover from eucaliptus to Pinus radiata. Agricultural and Forest Meteorology, 2000, vol. 100 , p. $59-72$.

(12) HUBER, A. \& A. IROUME. Variability of annual rainfall partitioning for different sites and forest covers in Chile. Journal of Hydrology 2001, $N^{\circ}$ 248, p. 78-92.

(13) HUBER, A., D. LÓPEZ. Cambios en el balance hídrico provocados por la tala rasa de un rodal adulto de Pinus radiata (D. Don). Bosque, 1993, vol. 14, $\mathrm{N}^{\circ} 2$, p. 11-18.

(14) NOVOA, P. Estimación de la evapotranspiración actual en bosques: teoría. Bosque, 1998, vol. 19, No 1, p. 111-121.

(15) FARAHANI, H., D. PETERSON, L. WESFALL, \& L. AHUJA. Soil water dry land cropping systems: The significance of cropping intensification. Soil Science Society of American Journal, 1998, vol. 62, p. 984-991.

(16) HUBER, A. y R. TRECAMAN. El efecto de las características de una plantación de Pinus radiata (D. Don) en la distribución espacial del contenido de agua edáfica. Bosque, 2000, vol. 21, $\mathrm{N}^{\circ} 1$, p. 37-44.

(17) LOPEZ F. Restauración hidrológico forestal y control de la erosión. Ingeniería Ambiental. Tragsa-Tragsatec, Ministerio del Medio Ambiente, Ediciones Mundiprensa, Madrid, España, 1998, 851 p.

(18) BOSCH, J. \& J. HEWLETT. A review of catchment experiments to determine the effects of vegetativo changes on water yield and evapotranspiration. Journal of Hydrology, 1982, vol. 55, p. 3-22.

(19) DUNCAN, M., The impact of afforestation on smallcatchment hydrology in Moutere Hills, Nelson. Seminar on Land-use in relation on water quantity and quality. Nelson Catchment Board, New Zealand, 1980, p. 60-90.

(20) IROUME, A. y A. HUBER. Intercepción de las lluvias por cubiertas de bosques y efectos en los caudales de crecida en una cuenca experimental en Malalcahuello, IX Región, Chile. Bosque, 2000, vol. 21, No 1, p. 45-56.

(21) SHARDA, V. et al. Hydrological behaviour of the Nilgiri sub-watersheds as affected by bluegum plantations, part II. Monthly water balances at different rainfa11 and runoff probabilities. Journal of Hydrology, 1988, vol. $103, \mathrm{~N}^{\circ} 3-4$, p. 347-355.

(22) SINGH, V. Applied modeling in catchment hydrology. Water Resources Publication, Colorado, Estados Unidos, 1982, $563 \mathrm{p}$.

(23) WRIGHT, K.,K. SENDEC, R. RICE \& R. THOMAS. Logging effects on streamflow: storm runoff at Caspar Creek in Northwestern California. Water Resources Research, 1990, vol. 26, $\mathrm{N}^{\mathrm{o}} 7$, p. 1.657-1.667.

(24) FAHEY, B., The effects plantation forestry on water yield in New Zealand. New Zealand Forestry, 1994, vol. 39, p. 18-23.

(25) MEUNIER, M., La cubierta forestal y las crecidas en las pequeñas cuencas de montaña. Revista Unasylva, 1996, vol. $47, \mathrm{~N}^{\circ} 185$, p. 29-37.

(26) DUNCAN, M., Does planting pinus reduce groundwater recharge?. New Zealand Hydrogical Society Symposium, Nelson, 1993, (No publicado).

(27) AYER, G., Reforestation with conifers; its effect on streamflow in Central New York. Water Resources Bulletin, 1968, vol 4, $\mathrm{N}^{\circ} 2$, p. 13.

(28) BERG, N., R. OSTERHUBER \& J. BERGMAN. Raininduced outflow from deep snow packs in the central Sierra Nevada, California. Department of Agriculture, Forest Service of the United States. Hydrological Sciences Journal, 1991, vol. 36, N 6, p. 611-629.

(29) BLACK, P. Watershed hydrology, Prentice Hall, New Jersey, Estados Unidos, 1991, 408 p.

(30) GIUPPONI, C. Tillage system effects on runoff and soil losses. Rivista di Ingegneria Agraria, 1993, vol. 24, $\mathrm{N}^{\circ} 4$, p. 224-253.

(31) REINHART, K., A. ESCHNER \& G. TRIMBLE. Effects on streamflow of four forest practices in the mountains of West Virginia. U. S. Forest Services Research Paper, Washington, Estados Unidos, 1963, NE-1, 79 p.

(32) RODIER, J. Caracteres generaux de l'hydrologie superficelle des zones arides et semi-arides en Afrique-leurs consequences sur les etudes des ingenieurs. Service Hydrologique, ORSTOM, Francia, 1990, 19 p.

(33) URIBE, H., J.L. ARUMI, L. GONZALEZ y L. SALGADO. Balances hidrológicos para estimar recarga de acuíferos en el secano interior, Chile. Ingeniería Hidráulica en México, 2003, vol. XVIII, No 3, p. 17-28.

(34) MENDELHALL, W. \& J. REINMUTH. Estadística para Administración y Economía. Grupo editorial Iberoamericana, Tercera ed., México, 1981, 707 p.

(35) APARICIO, F. Fundamentos de Hidrología de Superficie. Editorial Limusa, México, 1997, 303 p.

(36) PIZARRO, R., C. RAMIREZ y J.P. FLORES. Análisis comparativo de cinco métodos para la estimación de precipitaciones areales anuales en períodos extremos. Bosque, 2003, vol. 24, No 3, p. 31-38.

(37) CHOW, V., D. MAIDMENT \& D. MAYS. Hidrología Aplicada. Editorial Mc Graw-Hill, Santa Fe de Bogotá, Colombia, 1994, 584 p.

(38) UNESCO. ROSTLAC, Guía metodológica para la elaboración del balance hídrico de América del Sur. Oficina Regional y Tecnológica de la UNESCO para América Latina y el Caribe. Uruguay, 1982, 130 p. 